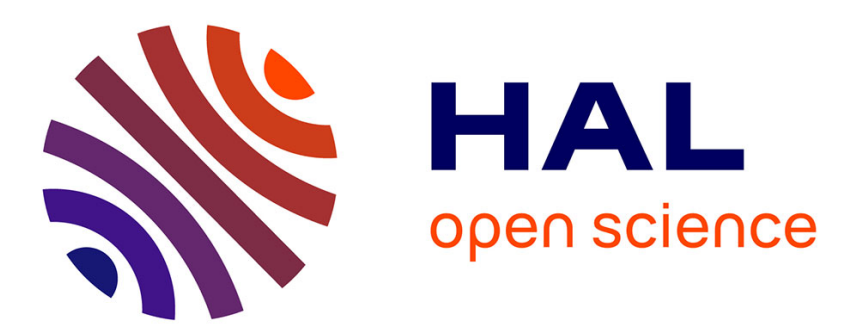

\title{
Hétérojonctions ZnTe/GaAlSb préparées par épitaxie en phase liquide
}

A. Joullié, A. Rossi, J.-F. Bresse, A. Jalil, V. Thierry-Mieg, Jacques Chevallier

\section{To cite this version:}

A. Joullié, A. Rossi, J.-F. Bresse, A. Jalil, V. Thierry-Mieg, et al.. Hétérojonctions ZnTe/GaAlSb préparées par épitaxie en phase liquide. Revue de Physique Appliquée, 1981, 16 (5), pp.209-216. 10.1051/rphysap:01981001605020900 . jpa-00244913

\section{HAL Id: jpa-00244913 https://hal.science/jpa-00244913}

Submitted on 1 Jan 1981

HAL is a multi-disciplinary open access archive for the deposit and dissemination of scientific research documents, whether they are published or not. The documents may come from teaching and research institutions in France or abroad, or from public or private research centers.
L'archive ouverte pluridisciplinaire HAL, est destinée au dépôt et à la diffusion de documents scientifiques de niveau recherche, publiés ou non, émanant des établissements d'enseignement et de recherche français ou étrangers, des laboratoires publics ou privés. 


\title{
Hétérojonctions ZnTe/GaAISb préparées par épitaxie en phase liquide
}

\author{
A. Joullie, A. Rossi (*), J.-F. Bresse (*), A. Jalil (**), V. Thierry-Mieg (**) et J. Chevallier (**) \\ Centre d'Etudes d'Electroniques des Solides ( ${ }^{1}$ ) \\ Université des Sciences et Techniques du Languedoc, 34060 Montpellier Cedex, France
}

(*) Laboratoire de Microscopie Electronique et de Microanalyse,

(**) Laboratoire de Physique des Solides, C.N.R.S., 1, place Aristide-Briand, F-92190 Meudon-Bellevue, France

(Reçu le 21 juillet 1980, révisé le 11 février 1981, accepté le 16 février 1981)

\begin{abstract}
Résumé. - Des hétérojonctions (p) $\mathrm{ZnTe} /(\mathrm{n}) \mathrm{Ga}_{1-x} \mathrm{Al}_{x} \mathrm{Sb}$ ont été obtenues par épitaxie en phase liquide de $\mathrm{Ga}_{1-x} \mathrm{Al}_{x} \mathrm{Sb}$ dopé au tellure sur substrat $\mathrm{ZnTe}$ dopé au phosphore, la concentration en AlSb des couches épitaxiées variant de 0 à 0,60 . La présence, à l'interface substrat-dépôt, d'un alliage quinaire $\left(\mathrm{Ga}_{1-x} \mathrm{Al}_{x}\right)_{1-y} \mathrm{Zn}_{y} \mathrm{Sb}_{1-z} \mathrm{Te}_{z}$ a été mise en évidence par analyse des structures à l'aide d'une microsonde électronique de Castaing. Les couches épitaxiées, de type n, sont fortement dopées au tellure (de l'ordre de $1 \%$ atomique). Une diffusion des éléments Ga, $\mathrm{Al}$ et $\mathrm{Sb}$ du dépôt dans le substrat de $\mathrm{ZnTe}$ a été détectée par S.I.M.S. sur une profondeur d'environ $10 \mu$. Cette diffusion rend le $\mathrm{ZnTe}$ semi-isolant, et luminescent dans le rouge. L'analyse des caractéristiques courant-tension à diverses températures laisse supposer que le courant direct est un courant de recombinaison de trous excités thermiquement dans la bande de valence. L'étude de la réponse spectrale montre que seuls les porteurs minoritaires du côté de $\mathrm{ZnTe}$ participent à la conduction sous éclairement. Une structure de bandes d'énergie est proposée pour une hétérojonction (p) $\mathrm{ZnTe} /(\mathrm{n}) \mathrm{Ga}_{0,40} \mathrm{Al}_{0,60} \mathrm{Sb}$.
\end{abstract}

Abstract. - Heterojunctions (p) $\mathrm{ZnTe} /(\mathrm{n}) \mathrm{Ga}_{1-x} \mathrm{Al}_{x} \mathrm{Sb}$ were grown by liquid phase epitaxy of $\mathrm{Te}$ doped $\mathrm{Ga}_{1-x} \mathrm{Al}_{x} \mathrm{Sb}$ onto $\mathrm{P}$ doped $\mathrm{ZnTe}$ substrates, with $x$ varying from 0 to 0.60 . The presence, at the substrate-deposite interface, of a quinary alloy $\left(\mathrm{Ga}_{1-x} \mathrm{Al}_{x}\right)_{1-y} \mathrm{Zn}_{y} \mathrm{Sb}_{1-z} \mathrm{Te}_{z}$, was shown by electron microprobe analysis. The epilayers were $\mathrm{n}$-type and heavily tellurium doped. A diffusion of $\mathrm{Ga}, \mathrm{Al}$ and $\mathrm{Sb}$ elements into the $\mathrm{ZnTe}$ substrate was detected by S.I.M.S. on about $10 \mu$. The diffused zones are semi-insulating and red cathodoluminescent. From the temperature dependence of the forward $I-V$ characteristics, we deduce that a space-charge recombination current could explain the conduction process in the dark. The spectral response of these heterojunctions showed that only the minority photocarriers created in $\mathrm{ZnTe}$ are collected. A band structure is proposed relative to a (p) $\mathrm{ZnTe} /(\mathrm{n}) \mathrm{Ga}_{0.40} \mathrm{Al}_{0.60} \mathrm{Sb}$ heterojunction.

1. Introduction. - Le composé ZnTe est un semiconducteur de type $p$ possédant une énergie de transition de bande interdite égale à $2,26 \mathrm{eV}$ à température ambiante. Il pourrait constituer une diode électroluminescente émettant dans le visible, s'il n'était pas très difficile à obtenir en type $\mathbf{n}$ par suite d'un phénomène d'autocompensation [1], d'où l'intérêt des hétérojonctions utilisant un semiconducteur de type $n$. Ainsi plusieurs travaux ont été publiés sur les hétérojonctions $\mathrm{ZnTe} / \mathrm{Si}$ [2], ZnTe/(II-VI) [3-6] et $\mathrm{ZnTe} /$ (III-V) [7-12].

La solution solide $\mathrm{Ga}_{1-x} \mathrm{Al}_{x} \mathrm{Sb}$ possède le même type de structure cristalline que $\mathrm{ZnTe}$, avec un paramètre de maille très proche de celui du ZnTe. Le

(1) Associé au C.N.R.S. (LA 21). désaccord de paramètre passe de $+0,12 \%$ avec $\mathrm{GaSb}$ à $-0,54 \%$ avec AlSb. Il est nul avec les alliages à $x=0,19$. Le matériau $\mathrm{Ga}_{1-x} \mathrm{Al}_{x} \mathrm{Sb}$ apparaît donc comme idéal pour la réalisation d'hétéroépitaxies avec $\mathrm{ZnTe}$. Par ailleurs, on sait que cet alliage est susceptible d'applications dans la conversion photovoltaïque de l'énergie solaire [13] et dans les systèmes rapides de communication par fibre optique centrés à $1,3-1,6 \mu \mathrm{m}$ [14-16]. Son énergie gap varie à $300 \mathrm{~K}$ de $0,70 \mathrm{eV}$ (transition directe, GaSb) à $1,60 \mathrm{eV}$ (transition indirecte, AlSb). Dans le couple $\mathrm{ZnTe} / \mathrm{GaAlSb}$, le $\mathrm{ZnTe}$ peut donc jouer le rôle d'une fenêtre optique et la solution solide celui du matériau absorbant. Cet article précise une méthode de préparation par épitaxie en phase liquide d'hétérojonctions (p) $\mathrm{ZnTe} /(\mathrm{n}) \mathrm{Ga}_{1-x} \mathrm{Al}_{x} \mathrm{Sb}$. Par analyse à la microsonde de Castaing, nous avons approché la structure à 
l'interface et détaillé la répartition des éléments à proximité de l'interface. Les résultats de l'étude des propriétés électriques des jonctions obtenues sont indiqués.

2. Croissance épitaxique. - Il existe deux méthodes possibles pour la réalisation d'hétérojonctions (p) $\mathrm{ZnTe} /(\mathrm{n}) \mathrm{GaAlSb}$ par épitaxie en phase liquide (E.P.L.) : croissance de $\mathrm{ZnTe}$ sur des couches de $\mathrm{GaAlSb}(\mathrm{n})$ épitaxiées sur substrat $\mathrm{GaSb}(\mathrm{n})$ ou croissance de $\mathrm{GaAlSb}(\mathrm{n})$ sur substrat $\mathrm{ZnTe}$.

La première méthode nécessite une double épitaxie et impose l'utilisation de solvants comme le zinc ou le tellure ou bien de solvants métalliques tels $\mathrm{Bi}, \mathrm{Pb}, \mathrm{Sn}$, $\mathrm{Ga}, \mathrm{In}$ [17] ou encore d'halogénures comme $\mathrm{ZnCl}_{2}$ [18]. Ces solvants présentent divers inconvénients : forte tension de vapeur (cas du $\mathrm{Zn}, \mathrm{Te}, \mathrm{ZnCl}_{2}$ ), introduction excessive du solvant dans le dépôt (cas des autres solvants). Nous avons opté pour la deuxième méthode en épitaxiant l'alliage $\mathrm{Ga}_{1-x} \mathrm{Al}_{x} \mathrm{Sb}$ dopé au tellure directement sur un substrat de $\mathrm{ZnTe}$, à partir d'un solvant gallium.

Le dispositif de croissance est un bâti classique d'E.P.L. qui utilise la technique du tiroir horizontal.

Le substrat est une pastille de ZnTe monocristalline, orientée (111) face Te, de type $p$, débitée dans un lingot Bridgman dopé au phosphore. Après polissage mécanique à $1 \mu$ chaque pastille est décapée dans une solution de brome-méthanol à $1,5 \%$ juste avant son introduction dans le réacteur.

La solution $(5 \mathrm{~g})$ est réalisée à partir des éléments gallium et aluminium (pureté $6 \mathrm{~N}$ ) alors que l'antimoine et le tellure sont introduits sous forme de pastilles de $\mathrm{GaSb}$ dopées $\mathrm{Te}$ de concentration en porteur de charge connue $\left(10^{18} \mathrm{~cm}^{-3}\right)$. La quantité de $\mathrm{GaSb}$ dopée est ajustée de façon à avoir une concentration en tellure dans le liquide

$$
X_{\mathrm{Te}}^{\mathrm{L}}=7 \times 10^{-7} \text { fr. at. }
$$

Nous verrons que cette valeur de concentration en $\mathrm{Te}$ peut être fortement augmentée par suite de la dissolution du substrat qui précède l'épitaxie proprement dite.

La solution est sous-saturée au départ en antimoine (de 0,5 à 1 at. \%). Elle est saturée ensuite à $500^{\circ} \mathrm{C}$ par dissolution d'une charge de $\mathrm{GaSb}$ non dopé. Cette saturation s'effectue pendant $40 \mathrm{~min}$. Le substrat de $\mathrm{ZnTe}$ est mis en contact du liquide une fois la charge retirée et la température du bain abaissée d'une valeur $\Delta$. La croissance s'effectue avec une vitesse de refroidissement $R$ uniforme.

Des couches de $\mathrm{Ga}_{1-x} \mathrm{Al}_{x} \mathrm{Sb}$ fortement dopées au tellure, de type $\mathrm{n}$ et de concentration $x$ dans la gamme $0<x<0,60$ ont été épitaxiées avec différentes valeurs de $R\left(10 ; 40 ; 74^{\circ} \mathrm{C} / \mathrm{h}\right.$ et différentes valeurs de la sursaturation initiale $\Delta\left(\right.$ de 0 à $\left.30^{\circ} \mathrm{C}\right)$. Pour un intervalle de croissance $\Delta T_{\mathrm{c}}$ de $15^{\circ} \mathrm{C}$, l'épaisseur des couches à $x=0,20$ est de $50 \mu$ avec $\Delta=3^{\circ} \mathrm{C}$ et $R=40^{\circ} \mathrm{C} / \mathrm{h}$. Cette épaisseur varie peu quand simul- tanément $\Delta$ et $R$ augmentent. Par contre, elle diminue lorsque $x$ croît $(20 \mu$ pour $x=0,60)$.

Le problème majeur de la croissance épitaxique de $\mathrm{Ga}_{1-x} \mathrm{Al}_{x} \mathrm{Sb}$ sur $\mathrm{ZnTe}$ a été la rapide dissolution $\mathrm{du}$ substrat de $\mathrm{ZnTe}$ dès qu'il est mis au contact de la solution liquide.

Ainsi, lorsque la croissance s'effectue à l'équilibre ou à partir de bains faiblement saturés $\left(\Delta T=3^{\circ} \mathrm{C}\right)$, l'interface substrat-dépôt n'est pas plane. En effet, le substrat de ZnTe se dissout au contact du bain ternaire, et la dissolution n'est pas régulière pour peu que le substrat soit désorienté. Cette dissolution, généralement en dentelures, provoque l'apparition d'inclusions de solvant à l'interface. Pour une saturation initiale de $3^{\circ} \mathrm{C}$, la zone perturbée à l'interface s'étend sur une profondeur de l'ordre de $3 \mu \mathrm{m}$.

Lorsque la croissance s'effectue à partir de bains fortement sursaturés $\left(\Delta T=20\right.$ à $\left.30^{\circ} \mathrm{C}\right)$, l'interface est rectiligne, sauf par endroits où le substrat a été dissous. Cette dissolution locale peut s'interpréter en supposant que la sursaturation imposée est supérieure à la sursaturation critique $\Delta T_{\mathrm{cr}}$ qui correspond à l'apparition de la nucléation de germes solides dans le volume du liquide. Dans ces conditions, il se forme autour de ces noyaux des zones liquides de composition proche de celle du liquidus et donc pour lesquelles il n'y a plus sursaturation. Les endroits du substrat au contact de ces zones sont alors dissous sur 1 à $2 \mu \mathrm{m}$.

Avec des sursaturations de $10^{\circ}-15^{\circ} \mathrm{C}$, l'interface substrat-dépôt est régulière, sans inclusion de solvant visible au microscope (Fig. 1).

La position de la jonction $p-n$ a été déterminée au Microscope Electronique à Balayage, par étude du courant induit sous l'effet du bombardement électro-

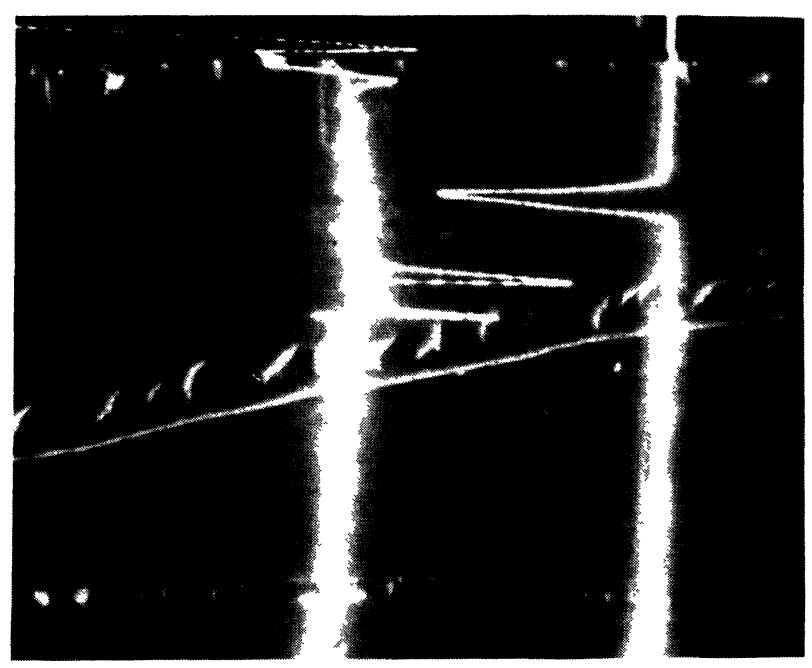

Fig. 1. - Dépôt de $\mathrm{Ga}_{1-x} \mathrm{Al}_{x} \mathrm{Sb}$ sur $\mathrm{ZnTe}$ obtenu avec comme conditions : $\Delta=15^{\circ} \mathrm{C} ; R=74^{\circ} \mathrm{C} / \mathrm{h} ; \Delta T_{\mathrm{c}}=20^{\circ} \mathrm{C} ; X_{\mathrm{Al}}^{\mathrm{L}}=0,035 \mathrm{fr}$. at. (image électronique avec enregistrement du courant induit par E.B.I.C. surimposé).

[ $\mathrm{Ga}_{1-x} \mathrm{Al}_{x} \mathrm{Sb}$ deposited on $\mathrm{ZnTe}$ with : $\Delta=15^{\circ} \mathrm{C} ; R=74^{\circ} \mathrm{C} / \mathrm{h}$; $\Delta T_{\mathrm{c}}=20^{\circ} \mathrm{C} ; X_{\mathrm{Al}}^{\mathrm{L}}=0.035$ at. fr. (Secondary electron image and E.B.I.C. profile superimposed.)] 
nique (E.B.I.C.). Dans tous les cas, elle est confondue avec l'interface dépôt-substrat, dont elle épouse la géométrie lorsque le substrat a été irrégulièrement dissous (Fig. 2).

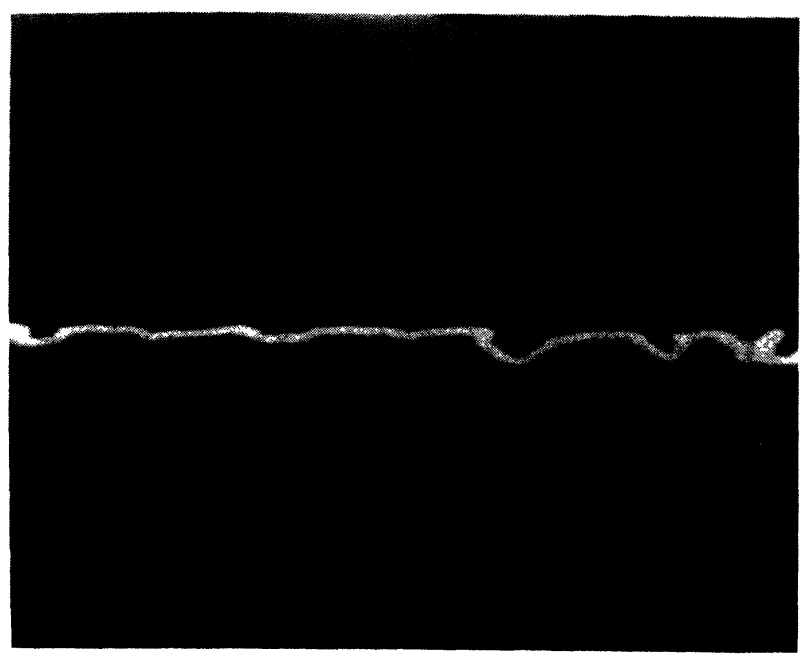

Fig. 2. - Visualisation de l'hétérojonction par E.B.I.C. pour une structure (n) $\mathrm{Ga}_{1-x} \mathrm{Al}_{x} \mathrm{Sb} /(\mathrm{p}) \mathrm{ZnTe}$ obtenue avec comme conditions : $\Delta=30^{\circ} \mathrm{C} ; R: 40^{\circ} \mathrm{C} / \mathrm{h} ; \Delta T_{\mathrm{r}}=15^{\circ} \mathrm{C} ; X_{\mathrm{A} 1}^{\mathrm{L}}=0,0125 \mathrm{fr}$. at.

[Visualisation of the heterojunction by E.B.I.C. for a (n) $\mathrm{Ga}_{1-x} \mathrm{Al}_{x} \mathrm{Sb} /(\mathrm{p}) \mathrm{ZnTe}$ structure obtained with : $\Delta=30^{\circ} \mathrm{C}$; $R=40^{\circ} \mathrm{C} / \mathrm{h} ; \Delta T_{\mathrm{c}}=15^{\circ} \mathrm{C}: X_{\mathrm{A} 1}^{\mathrm{L}}=0.0125$ at. fr. $]$

3. Profils de composition. - 3.1 ZONE D'INTERFACE SUBSTRAT-DÉPÔT. - L'étude de la composition des couches épitaxiées a été réalisée à l'aide d'une sonde électronique de Castaing sur des tranches biseautées à $3^{\circ}$, en utilisant de faibles valeurs de tension du faisceau primaire (6 et $15 \mathrm{keV}$ ) ceci afin de réduire la profondeur de pénétration des électrons et le volume ionisé. Deux exemples de répartition des cinq éléments $\mathrm{Ga}, \mathrm{Al}, \mathrm{Sb}, \mathrm{Zn}$ et $\mathrm{Te}$ sont présentés Figures 3 et 4.

Les analyses confirment de précédentes études d'hétéroépitaxies du type (II-VI)/(III-V) [7, 9, 11, 12, $20,21]$ qui ont montré la présence à l'interface substratdépôt d'une zone intermédiaire complexe, supposée être une solution solide entre le binaire (II-VI) et le binaire (III-V). Cette zone intermédiaire est caractérisée ici par des variations brutales de compositions, sur des distances de l'ordre du micron, telles que :

et

$$
x_{\mathrm{Ga}}^{\mathrm{s}}+x_{\mathrm{A} 1}^{\mathrm{s}}+x_{\mathrm{Zn}}^{\mathrm{s}}=0,50 \text { fr. at. }
$$

$$
x_{\mathrm{Sb}}^{\mathrm{s}}+x_{\mathrm{Te}}^{\mathrm{s}}=0,50 \text { fr. at. }
$$

On en déduit que le tellure se substitue à l'antimoine et que le zinc se substitue au gallium et à l'aluminium.

Près de cette zone d'interface, on note une augmentation de la concentration en aluminium, d'où un léger pic qui tend à disparaître quand le pourcentage en AlSb augmente, et dont la valeur, déterminée d'après le rapport $\mathrm{Al} / \mathrm{Ga}=x / 1-x$, correspond à celle déduite du diagramme de phases du système

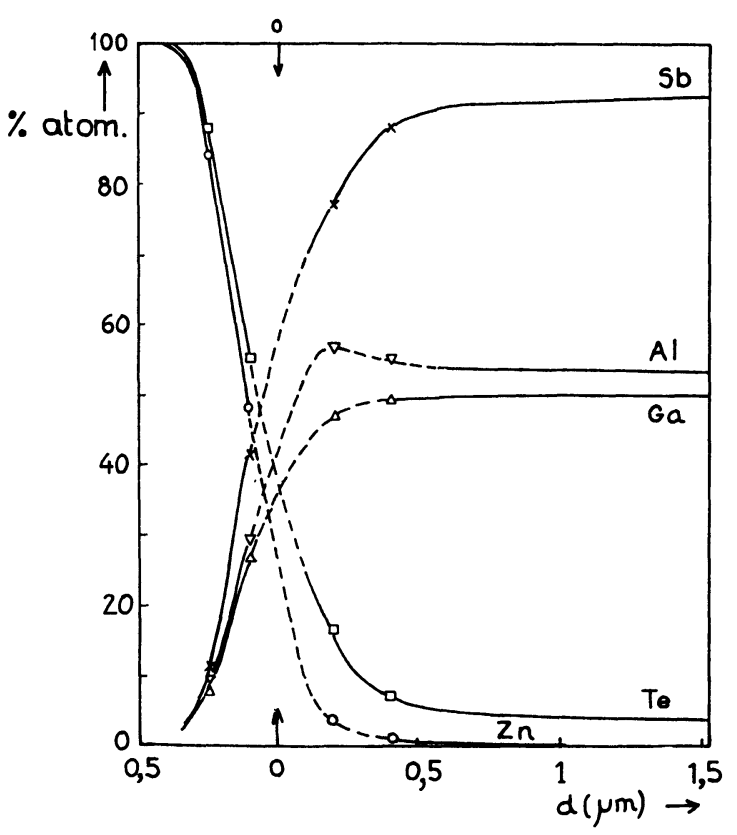

Fig. 3. - Profils de concentration en éléments $\mathrm{Ga}, \mathrm{Al}, \mathrm{Sb}, \mathrm{Zn}, \mathrm{Te}$, à l'interface substrat-dépôt d'une épitaxie réalisée avec $\Delta=20^{\circ} \mathrm{C}$; $R=74^{\circ} \mathrm{C} / \mathrm{h} ; X_{\mathrm{Al}}^{\mathrm{L}}=0,026 \mathrm{fr}$. at. Les courbes en pointillés correspondent aux endroits où le volume d'excitation de l'élément analysé pénètre à la fois dans le dépôt et dans le substrat. Les points et triangles sont relatifs à des mesures de concentration effectuées sonde fixe et déterminées à partir du programme de corrections MAGIC [26].

[Concentration profiles of $\mathrm{Ga}, \mathrm{Al}, \mathrm{Sb}, \mathrm{Zn}, \mathrm{Te}$ elements at the substrate-deposited layer interface of an epitaxy performed with : $\Delta=20^{\circ} \mathrm{C} ; R=74^{\circ} \mathrm{C} / \mathrm{h} ; X_{\mathrm{Al}}^{\mathrm{L}}=0.026$ at. fr. Dashed curves are in the place where the excitation volume of the analysed element enters both into the substrate and the deposited layer. Rounds and triangles are the results of quantitative measurements [26].]

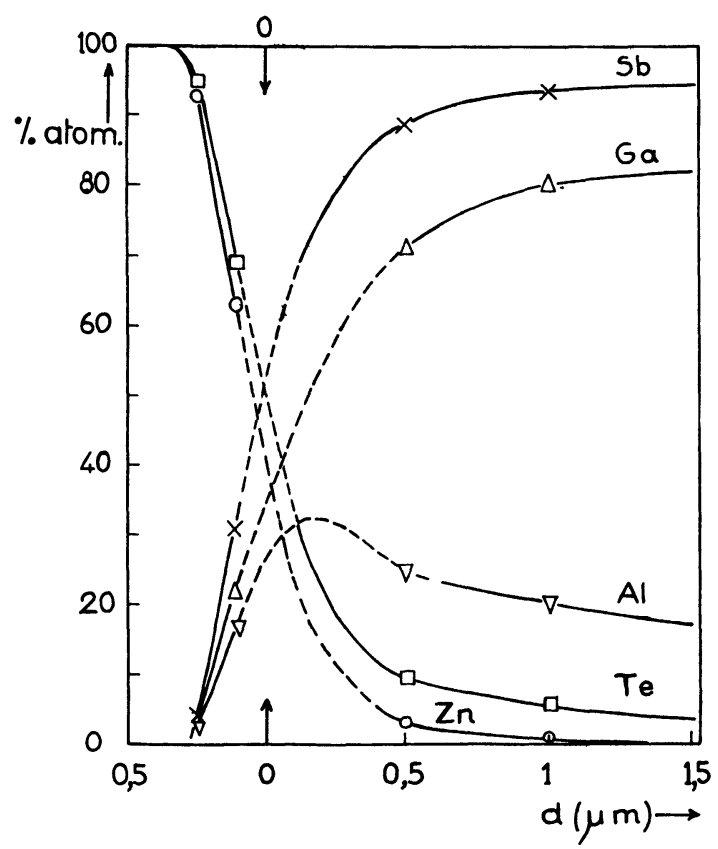

Fig. 4. - Profils de concentration en éléments à l'interface substratdépôt d'une épitaxie réalisée avec $\Delta=15^{\circ} \mathrm{C} ; R=74^{\circ} \mathrm{C} / \mathrm{h}$; $X_{\mathrm{A} 1}^{\mathrm{L}}=0,007 \mathrm{fr}$. at.

[Concentration profiles of elements at the substrate-deposite interface of an epitaxy performed with : $\Delta=15^{\circ} \mathrm{C} ; R=74^{\circ} \mathrm{C} / \mathrm{h}$; $X_{\mathrm{Al}}^{\mathrm{L}}=0.007$ at. fr.] 
Ga-Al-Sb [19, 22]. On a donc probablement dans la zone d'interface un alliage quinaire du type : $\left(\mathrm{Ga}_{1-x} \mathrm{Al}_{x}\right)_{1-y} \mathrm{Zn}_{y} \mathrm{Sb}_{1-z} \mathrm{Te}_{z}$.

3.2 COMPOSITION DU DÉPÔT. - La composition du dépôt, hors de la zone d'interface, varie faiblement le long de l'épaisseur du dépôt (Figs 5 et 6). La présence de zinc et de tellure a été mise en évidence dans la couche épitaxiée. Les quantités introduites sont fonction de la sursaturation initiale $\Delta$. Les dépôts obtenus avec $\Delta=3^{\circ} \mathrm{C}$ contiennent un peu de zinc ( $<1$ at. $\%$ ) et plusieurs at. $\%$ de tellure $(2-5$ at. $\%)$. Avec ceux obtenus à partir de $\Delta=15^{\circ} \mathrm{C}$, la teneur en zinc n'est plus mesurable à $3 \mu$ de l'interface, mais le tellure reste présent avec une forte concentration $(0,5-1$ at. \%). Ce résultat indique que la dissolution du substrat de $\mathrm{ZnTe}$ est réduite, mais pas complètement éliminée lorsque l'épitaxie se réalise avec des sursaturations considérées comme optimales $\left(10-15^{\circ} \mathrm{C}\right)$, et d'autre part, que le coefficient de ségrégation du tellure est plus élevé que celui du zinc. Les analyses quantitatives montrent que la composition du dépôt est donnée par: $\mathrm{Ga}_{1-x} \mathrm{Al}_{x} \mathrm{Sb}_{1-z} \mathrm{Te}_{z}$.

3.3 ZONE DIFFUSÉE DU SUBSTRAT. - Une analyse de la répartition des divers éléments a été effectuée avec une sonde ionique, pour une structure $\mathrm{ZnTe} / \mathrm{Ga}_{0,4} \mathrm{Al}_{0,6} \mathrm{Sb}$ élaborée avec une sursaturation initiale de $15^{\circ} \mathrm{C}$. Deux cratères différents ont été

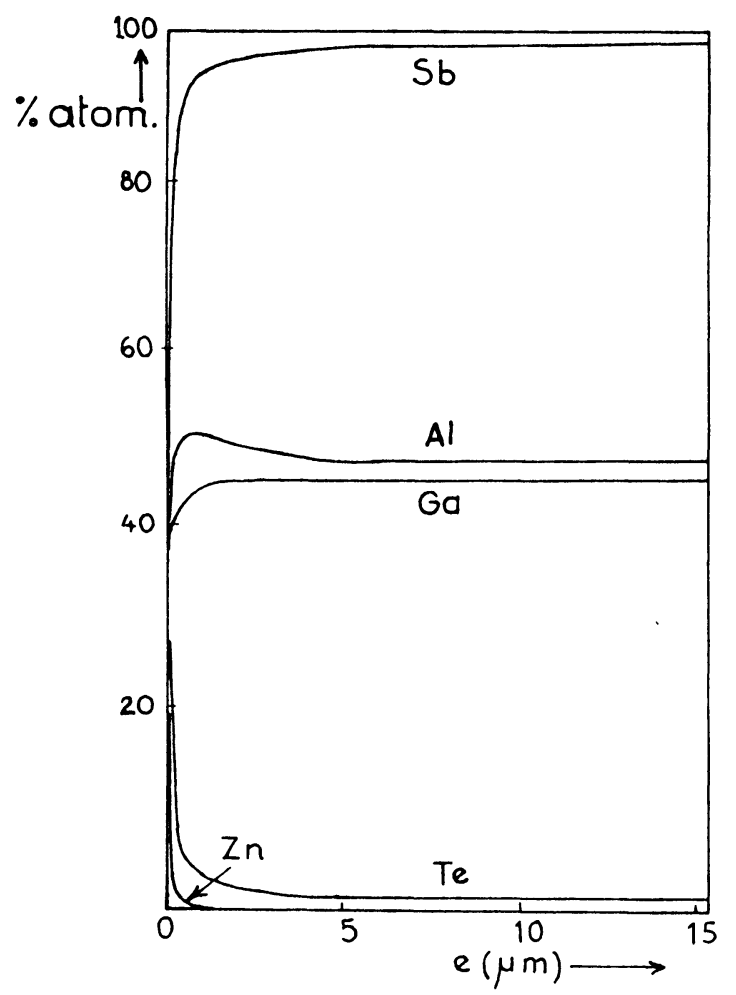

Fig. 5. - Profils de composition d'une couche épitaxiée dans les conditions indiquées figure 3 . La couche contient du tellure. Le zinc n'est plus décelé à la sonde électronique.

[Composition profiles of an epilayer obtained under the same conditions as indicated figure 3. The layer contains a few $\mathrm{Te}$, but $\mathrm{Zn}$ is not detected by electron microprobe analysis.]

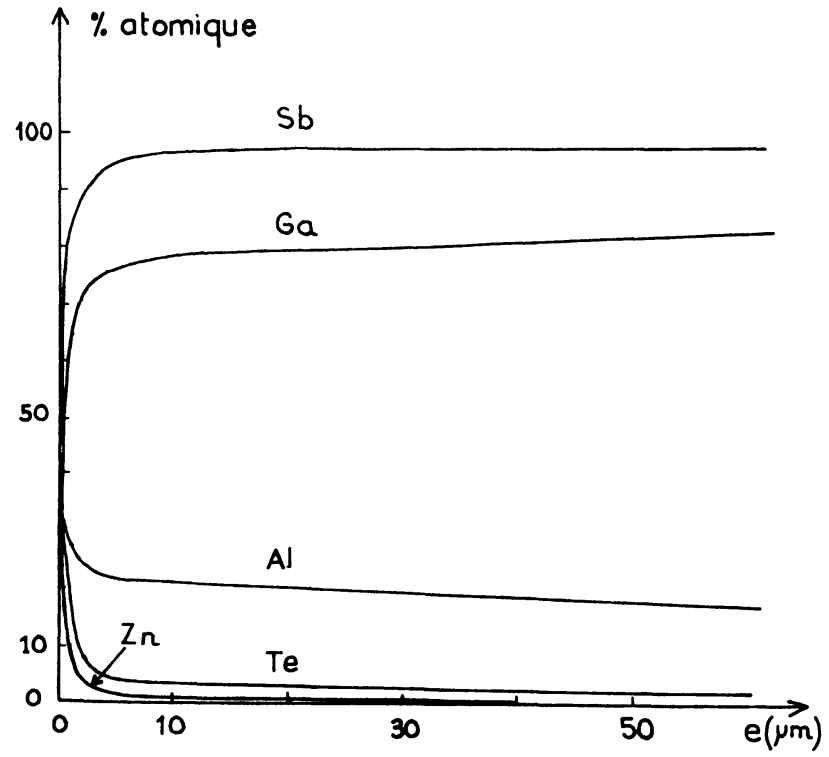

Fig. 6. - Profils de composition d'une couche épitaxiée avec $\Delta=3^{\circ} \mathrm{C} ; R=40^{\circ} \mathrm{C} / \mathrm{h} ; X_{\mathrm{Al}}^{\mathrm{L}}=0,007 \mathrm{fr}$. at. Les quantités importantes de $\mathrm{Zn}$ et Te présentes dans la couche proviennent de la dissolution initiale du substrat de $\mathrm{ZnTe}$.

[Composition profiles of a layer epitaxied with : $\Delta=3^{\circ} \mathrm{C}$; $R=40^{\circ} \mathrm{C} / \mathrm{h} ; X_{\mathrm{Al}}^{\mathrm{L}}=0.007$ at. fr. The great content of $\mathrm{Zn}$ and $\mathrm{Te}$ in the layer are due to the initial dissolution of the ZnTe substrate.]

formés, le premier pour $\mathrm{Ga}_{69}^{+}$et $\mathrm{Zn}_{64}^{+}$, le second pour $\mathrm{Al}_{27}^{+}, \mathrm{Te}_{128}^{+}$et $\mathrm{Sb}_{121}^{+}$. L'analyse confirme les résultats obtenus à la microsonde électronique, à savoir un dépôt à composition relativement uniforme contenant les éléments $\mathrm{Ga}, \mathrm{Al}, \mathrm{Sb}$ et $\mathrm{Te}$, et une zone d'interface dont l'épaisseur a été évaluée, selon le cratère, entre 1,5 et $3 \mu$.

Côté substrat, on observe une zone perturbée montrant un appauvrissement en $\mathrm{Zn}$ et Te sur environ $4 \mu$. Dans cette zone on enregistre la présence des trois éléments du dépôt $\mathrm{Ga}, \mathrm{Al}$ et $\mathrm{Sb}$. Au-delà (jusqu'à plus de $10 \mu$ ) ces éléments existent en beaucoup plus faible concentration. Il y a donc eu diffusion des éléments $\mathrm{Ga}, \mathrm{Al}$ et $\mathrm{Sb}$ en cours d'épitaxie.

Cette diffusion a pour effet de modifier la luminescence classique de bord de bande du ZnTe dopé phosphore qui de verte, devient jaune-rouge au voisinage de l'interface, sur une dizaine de microns. On sait que le $\mathrm{ZnTe}$ dopé $\mathrm{Al}$ émet dans le jaune et le $\mathrm{ZnTe}$ dopé $\mathrm{Ga}$ émet dans le rouge.

4. Caractéristiques électriques des hétérojonctions. - 4.1 RÉsultats. - Les substrats de ZnTe sont dopés au phosphore $\left(N_{\mathrm{A}}=10^{18}\right.$ at. $\left.\mathrm{cm}^{-3}\right)$ ce qui donne à $300 \mathrm{~K}$ une concentration en porteurs

$$
p \simeq 5 \times 10^{16} \mathrm{~cm}^{-3},
$$

une mobilité de Hall $\mu_{\mathrm{H}} \sim 70 \mathrm{~cm}^{2} \mathrm{~V}^{-1} \mathrm{~s}^{-1}$ et une résistivité $\rho=1 \Omega . \mathrm{cm}$. A $77 \mathrm{~K}$, ces valeurs passent respectivement à $\sim 10^{15} \mathrm{~cm}^{2} \cdot \mathrm{V}^{-1} \cdot \mathrm{s}^{-1}$ et $5 \Omega . \mathrm{cm}$.

Les couches épitaxiées de $\mathrm{Ga}_{1-x} \mathrm{Al}_{x} \mathrm{Sb}$ sont de type $n$, et par suite de la dissolution du substrat, dopées faiblement au zinc mais très fortement au 
tellure $(0,5$ à 1 at. \%). Les mesures d'effet Hall et de résistivité, effectuées par la méthode de Van Der Paw ont fourni à $300 \mathrm{~K}$ :

$$
\begin{aligned}
n & \sim 2 \times 10^{17} \mathrm{~cm}^{-3} ; \\
\mu_{\mathrm{H}} & \left.\sim 250 . \mathrm{V}^{-1} \cdot \mathrm{s}^{-1} \text { (échantillon à } X<0,17\right)
\end{aligned}
$$

et

$$
\begin{aligned}
\mu_{\mathrm{H}} & \sim 50 \mathrm{~cm} \cdot \mathrm{V}^{-1} \cdot \mathrm{s}^{-1} \quad(\text { échantillon à } X \geqslant 0,17) ; \\
p & \sim 0,1 \Omega \cdot \mathrm{cm} .
\end{aligned}
$$

Ces valeurs évoluent peu à $77 \mathrm{~K}$.

La longueur de diffusion des porteurs minoritaires a été déterminée de chaque côté de la jonction par la méthode E.B.I.C. On a trouvé $\mathrm{Ln} \sim \mathrm{Lp} \sim 0,5 \mu$. Les valeurs de $L p$ sont indépendantes de la composition de la couche épitaxiée.

Des mesures de courant-tension à l'obscurité et sous éclairement, de capacité tension et de réponses spectrales, ont été pratiquées sur des jonctions p-n de $15 \mathrm{~mm}^{2}$ de surface obtenues par clivage de la pastille initiale; les côtés non clivés sont rodés à l'aide d'alumine. Après un nettoyage au trichloréthylène chaud et à l'acétone chaud, la surface de GaAlSb étant protégée avec un vernis qui résiste parfaitement à l'alcool bromé, on décape la surface de $\mathrm{ZnTe}$ au brome méthanol. Aussitôt après, on réalise deux dépôts de platine par électrolyse d'une solution de platine sur la surface de $\mathrm{ZnTe}$. On dégage ensuite la surface de GaAlSb avec de l'acétone bouillant. Puis on fixe des fils d'or ou de platine avec des billes d'indium du côté de GaAlSb et sur les dépôts de platine côté $\mathrm{ZnTe}$ à l'aide d'un fer à souder de faible puissance. Ensuite, l'ohmicité des contacts est vérifiée à l'aide d'un diodoscope.

Un exemple type de variation de la capacité des hétérojonctions en fonction de la tension de polarisation est donné figure 7. La capacité varie peu avec la tension de polarisation. Ce genre de variation est caractéristique d'une couche intermédiaire semi-isolante. En effet, la diffusion des éléments de la

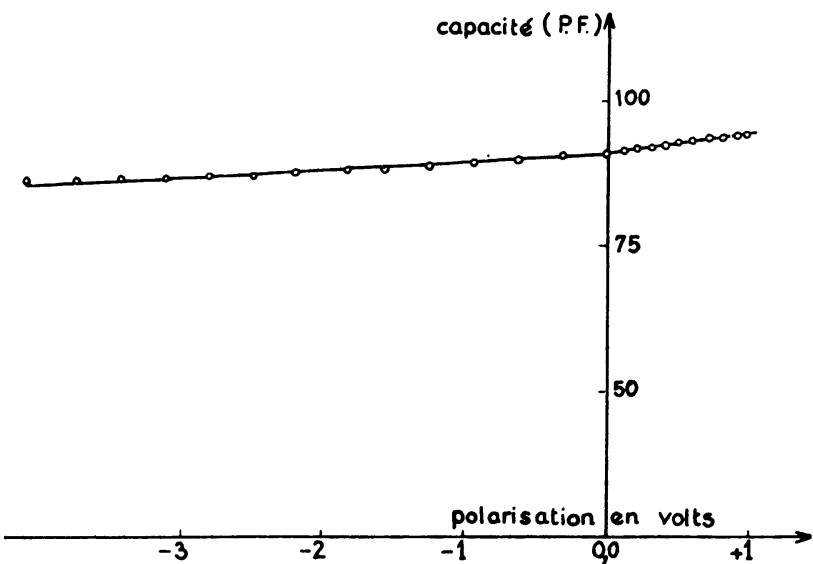

Fig. 7. - Variation de la capacité en fonction de la tension de polarisation.

[Variation of the capacitance versus the junction bias.] colonne III dans le $\mathrm{ZnTe}$ donne naissance à une région compensée de très grande résistivité [25]. L'épaisseur de cette couche semi-isolante a été évaluée pour la plupart des échantillons entre 10 et $15 \mu$, à partir de la valeur de $c$ à tension nulle et la constante diélectrique du $\mathrm{ZnTe}\left(\varepsilon=10,3 \varepsilon_{0}\right)$. Un tel résultat a été obtenu avec des hétérojonctions du type $\mathrm{ZnTe} /(\mathrm{III}-\mathrm{V})$ élaborées par E.P.L. [11] ou d'autres techniques [7, 8, 9]. La formation de cette couche semi-isolante a été interprétée par suite de la diffusion de l'élément III dans le substrat.

Les caractéristiques courant-tension des hétérojonctions ont été déterminées à différentes températures. Elles sont peu liées à la composition du dépôt. La figure 2 montre l'évolution des courbes $I-V$ sous polarisation directe en fonction de la température. On distingue trois régions :

- pour de faibles courants, les caractéristiques $I-V-T$ suivent une loi ohmique ;

- pour des courants élevés, les caractéristiques $I-V-T$ sont dominées par la résistance série du substrat (épaisseur de l'ordre du $\mathrm{mm}$ ) et de la couche ;

- une région intermédiaire où $I(V)$ peut être représenté par l'expression :

$$
I=I_{\mathrm{s}} \exp \left[\frac{q V}{n k T}\right]
$$

Le facteur d'idéalité $n$ augmente avec la température comme indiqué sur le tableau I ce qui traduit un comportement plus complexe qu'un phénomène de recombinaison dans une barrière symétrique. Nous avons trouvé que l'inverse de $n$ suit une loi linéaire en $T$ :

$$
\beta=1 / n=-a T+b
$$

avec

$$
a=0,266 \mathrm{k}^{-1} \cdot \mathrm{V}^{-1} \text { et } b=93,6 \mathrm{~V}^{-1}
$$

Les valeurs de $\beta$ sont également portées dans le tableau I.

Tableau I. - Variations de $\beta$ et du facteur de qualité n avec la température.

$\begin{array}{cccc}T(\mathrm{~K}) & \beta=\frac{\partial \ln I}{\partial V}\left(\mathrm{~V}^{-1}\right) & k T(\mathrm{eV}) & n=1 /\left[\beta \cdot\left(\frac{k T}{q}\right)\right] \\ \overline{297} & \overline{15,3} & -\overline{0256} & -\overline{2,55} \\ 264 & 23,3 & 0,0227 & 1,89 \\ 230 & 32,7 & 0,0198 & 1,54 \\ 175 & 47,2 & 0,0151 & 1,40 \\ 135 & 63,2 & 0,0116 & 1,36\end{array}$

L'évolution des caractéristique $I-V$ en polarisation inverse est précisée figure 9 en fonction de la température. Elle est de la forme $I=A V^{n}$. Pour de faibles courants, $n=1$. Cette région ohmique peut être attribuée, comme en polarisation directe, à un courant de fuite. Pour des valeurs de courant élevées, ces 


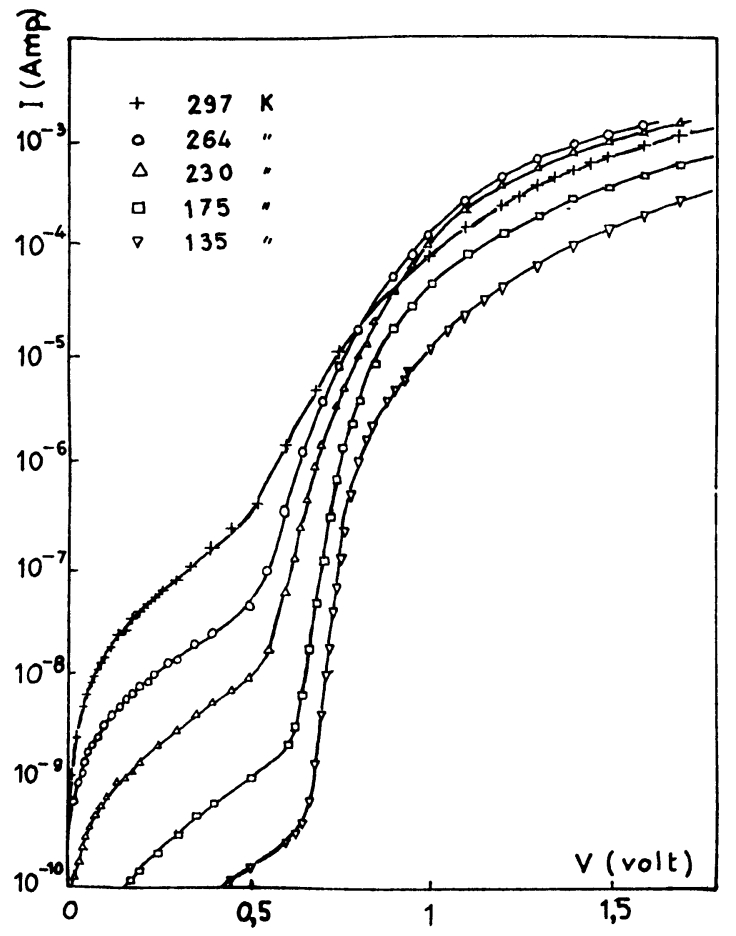

Fig. 8. - Caractéristiques courant-tension en polarisation directe pour différentes températures.

[Direct $I-V$ characteristics at different temperatures.]

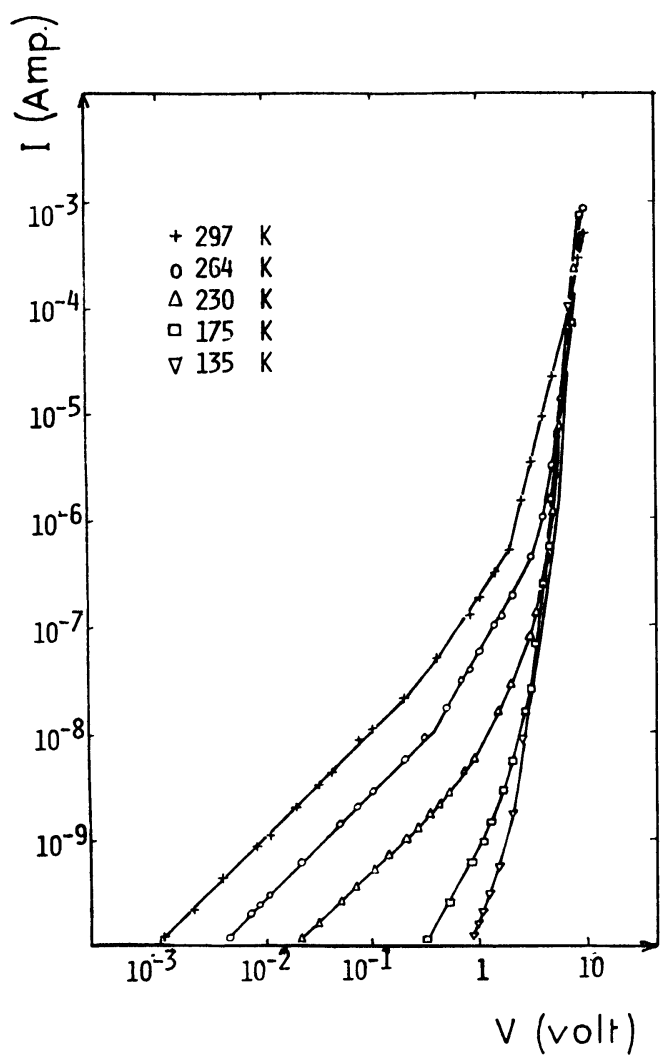

Fig. 9. - Caractéristiques courant-tension en polarisation inverse pour différentes températures.

[Reverse $I-V$ characteristics at different temperatures.] caractéristiques deviennent indépendantes de la température.

4.2 Discussion. - L'analyse des caractéristiques courant tension permet de préciser les mécanismes de conduction à l'obscurité. L'extrapolation des parties droites des caractéristiques $I(V)$ de la figure 8 donne la valeur de $I_{\mathrm{s}}$ à chaque température; ces valeurs sont reportées en fonction de l'inverse de la température sur la figure 10. La dépendance de $I_{\mathrm{s}}$ vis-à-vis de la température est :

$$
I_{\mathrm{s}}=I_{\mathrm{s} 0} \exp -\frac{E_{\mathrm{a}}}{k T}
$$

où $E_{\mathrm{a}}$ est l'énergie d'activation associée au mécanisme de conduction en polarisation directe; on trouve $E_{\mathrm{a}}=0,92 \mathrm{eV}$.

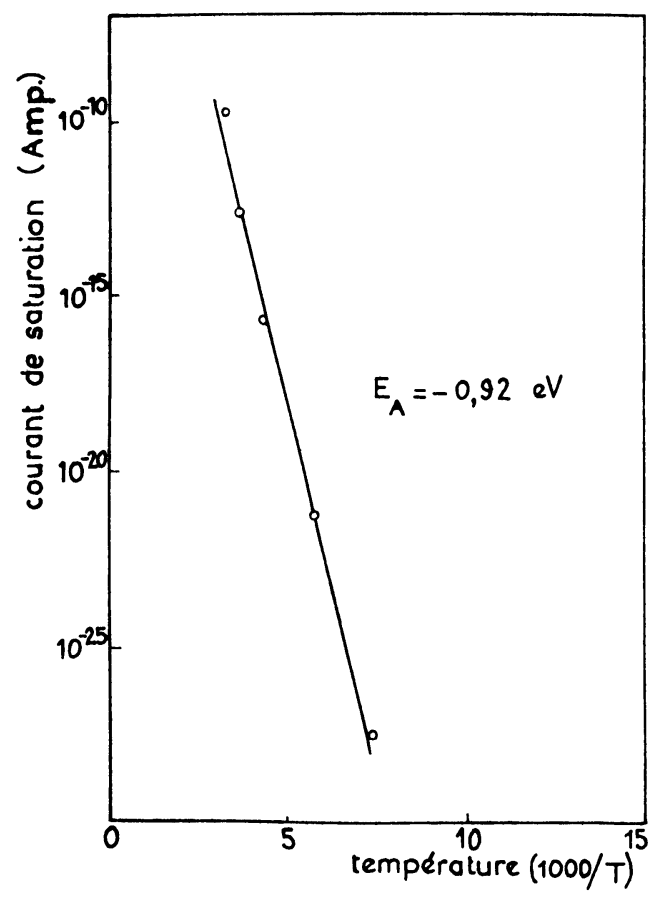

Fig. 10. - Variation du courant de saturation en fonction de l'inverse de la température.

[Variation of the saturation current versus $T^{-1}$.]

Par ailleurs, la valeur théorique maximale de $I_{\mathrm{s}}$ peut être estimée à partir de la relation

$$
I_{\mathrm{s}}=A T^{2} \exp \left(-\frac{q \varphi B}{k T}\right)
$$

exprimant le courant d'émission thermo-ionique audessus d'une barrière $q \varphi_{B n}, A$ étant la constante de Richardson modifiée. En prenant $A=60 \mathrm{~A} / \mathrm{cm}^{2} / \mathrm{K}^{2}$ et $q \varphi_{B n}=0,9 \mathrm{eV}$, nous trouvons un courant de $1,3 \times 10^{-9} \mathrm{~A} / \mathrm{cm}^{2}$ à température ambiante du même ordre de grandeur que la valeur expérimentale $I_{\mathrm{s}}$ à température ambiante $\left(\simeq 10^{-9} \mathrm{~A} / \mathrm{cm}^{2}\right)$. Nous concluons donc que le courant est limité par l'excitation de 


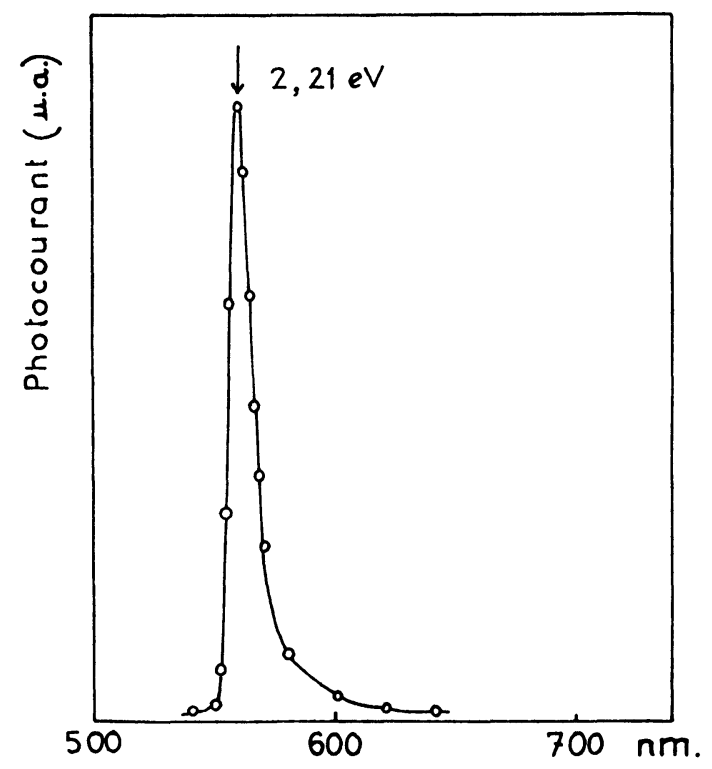

Fig. 11. - Réponse spectrale typique d'une hétérojonction.

[Typical spectral response of an heterojunction.]

porteurs au-dessus d'une barrière de hauteur $0,92 \mathrm{eV}$. Ces données permettent de proposer la structure de bandes d'énergie représentée sur la figure 12. A l'interface $\mathrm{GaAlSb} / \mathrm{ZnTe}$, les discontinuités entre les bandes de valence et les bandes de conduction ont été fixées de manière classique d'après les différences d'affinités électroniques et de largeurs de bande interdite. La barrière de hauteur $0,92 \mathrm{eV}$ déterminée ci-dessus doit donc apparaître du côté $\mathrm{ZnTe}$ entre la base conductrice et la couche intermédiaire isolante. La charge d'espace développée dans $\mathrm{ZnTe}$ est équilibrée par la

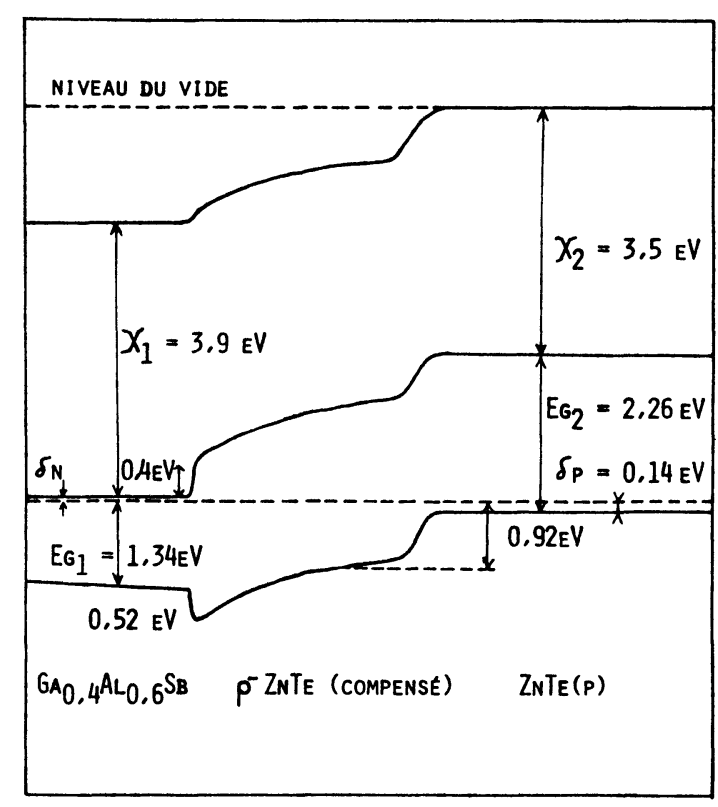

Fig. 12. - Exemple de structure de bandes d'énergies pour une hétérojonction (p) $\mathrm{ZnTe} /(\mathrm{n}) \mathrm{Ga}_{0,40} \mathrm{Al}_{0,60} \mathrm{Sb}$ préparée par Epitaxie en Phase Liquide.

[Exemple of an energy band diagram of a $(\mathrm{p}) \mathrm{ZnTe} /(\mathrm{n}) \mathrm{Ga}_{0.40} \mathrm{Al}_{0.60} \mathrm{Sb}$ heterojunction prepared by Liquid Phase Epitaxy.] charge positive apparaissant dans GaAlSb dopé au sein de la barrière de hauteur $0,4 \mathrm{eV}$. Le mécanisme limitant le courant en polarisation directe est ainsi l'excitation thermique de trous libres à partir de $\mathrm{ZnTe}$. Ces trous se recombinent ensuite rapidement au sein de la couche isolante avec des électrons provenant de GaAlSb. La répartition complexe et non connue du potentiel appliqué au sein de la structure est à l'origine des valeurs du facteur d'idéalité $\boldsymbol{n}$ variant avec la température et différant de la valeur théorique $n=2$.

5. Réponse spectrale des hétérojonctions. - Une réponse spectrale typique des hétérojonctions $\mathrm{ZnTe} /$ GaAlSb est représentée sur la figure 11. Elle a été obtenue par illumination du côté $\mathrm{ZnTe}$. On constate qu'elle se limite à un pic centré à 2,21 eV. Aucune réponse n'apparaît en particulier au voisinage de l'énergie de la bande interdite de l'alliage $(\simeq 1,34 \mathrm{eV})$. Ainsi, les photoporteurs créés dans GaAlSb ne sont pas collectés. Seuls sont recueillis les électrons du ZnTe. Plusieurs hypothèses peuvent être avancées :

- la présence du spike dans la bande de valence qui empêche le passage des trous;

- présence de pièges à l'interface.

Par ailleurs, la composition superficielle en $\mathrm{Al}$ est généralement inférieure à la composition interfaciale, ce qui favorise la création d'un gradient de bande interdite qui tend à entraîner les trous dans le $\mathrm{GaAlSb}$ vers la surface.

6. Conclusion. - Les résultats obtenus avec les hétérojonctions $\mathrm{ZnTe} / \mathrm{Ga}_{1-x} \mathrm{Al}_{x} \mathrm{Sb}$ préparées par Epitaxie en Phase Liquide sont dans leur ensemble comparables à ceux fournis par d'autres types d'hétérojonctions $\mathrm{ZnTe} /(\mathrm{III}-\mathrm{V})$. Il y a formation d'une zone d'interface entre le substrat de ZnTe et le dépôt de $\mathrm{Ga}_{1-x} \mathrm{Al}_{x} \mathrm{Sb}$, qui est une solution solide du type :

$$
\left(\mathrm{Ga}_{1-x} \mathrm{Al}_{x}\right)_{1-y} \quad \mathrm{Zn}_{y} \mathrm{Sb}_{1-z} \mathrm{Te}_{z} \text {. }
$$

Le dépôt est lui-même fortement dopé en tellure (0,5-1 at. \%), par suite de la dissolution inévitable du substrat qui se produit dès l'introduction de celui-ci dans la solution liquide. Par ailleurs, une diffusion des éléments du dépôt $\mathrm{Ga}, \mathrm{Al}$ et $\mathrm{Sb}$ dans $\mathrm{ZnTe}$ a été décelée à la sonde ionique pour un couple

$$
\mathrm{ZnTe} / \mathrm{Ga}_{0,40} \mathrm{Al}_{0,60} \mathrm{Sb} \text {. }
$$

Cette diffusion crée une région compensée de forte résistivité dans $\mathrm{ZnTe}$. Son épaisseur a été estimée par mesure $C-V$ à une dizaine de microns.

La position de la jonction a été visualisée au M.E.B. Elle est située dans la zone d'interface substrat-dépôt.

L'analyse des caractéristiques $I-V$ à l'obscurité, à différentes températures, montre que le courant direct est limité par l'excitation de trous libres dans la région compensée à partir du $\mathrm{ZnTe}$. Ces trous se recombinent 
ensuite avec les électrons en provenance de GaAlSb.

L'analyse des réponses spectrales montre que seuls les porteurs minoritaires du côté $\mathrm{ZnTe}$ participent à la création du photocourant.

Sur la figure 12 est présentée une structure possible de bandes d'énergie pour une hétérojonction $\mathrm{ZnTe} / \mathrm{Ga}_{0,40} \mathrm{Al}_{0,60} \mathrm{Sb}$, compatible avec les principales caractérisations effectuées.
Remerciements. - Nous tenons à remercier M. M. Schneider pour la fourniture des substrats de ZnTe, Mme C. Grattepain pour les analyses de sonde ionique et M. R. L. Aulombard pour la caractérisation électrique des couches épitaxiées. Nous adressons aussi nos remerciements à $M$. Y. Marfaing pour les discussions fructueuses que nous avons partagées avec lui.

\section{Bibliographie}

[1] Mandel, G., Phys. Rev. 134 (1964) A 1073.

[2] Kot, M. V. et Panasyuk, L. M., Sov. Phys. Semicond. 1 (1967) 155.

[3] Aven, M. et Garwacki, W., J. Electrochem. Soc. 110 (1963) 401.

[4] Tsujimoto, Y. et FukaI, M., Jpn. J. Appl. Phys. 6 (1967) 1013 et 6 (1967) 1024.

[5] Fujita, S., Morial, F., Arai, S. et Sakaguchi, T., Jpn. J. Appl. Phys. 12 (1973) 1841.

[6] Al Dallai, S., Thèse de Docteur Ingénieur, Paris VI (1976).

[7] Serreze, H., Fischler, S. et Sawyer, D., J. Appl. Phys. 39 (1968) 5330.

[8] Takahashi, K., Baker, W. D. et Milnes, A. G., Int. J. Electron. 27 (1969) 383.

[9] Nakai, J., Kamuro, S., Fukushima, M. et Hamguchi, C., Proc. Int. Conf. Phys. Chem. heterojunctions, Budapest.

[10] Morizumi, T., Ota, T. et Takahashi, K., Jpn. J. Appl. Phys. $9(1970) 849$ et $10(1971) 1652$.

[11] Tamura, T., Morizumi, T. et Takahashi, K., Jpn. J. Appl. Phys. 10 (1971) 813 et 11 (1972) 1024.

[12] Ota, T., Kinoshita, H. et TaKahashi, K., Electr. Eng. in Jpn

[13] Nguyen Van Mau, A., Muoy, Y., Bougnot, G., Bresse, J.-F. et Moussali, G. M., Photovoltaic Solar Energy Conference, Luxembourg, 27-30 sept. (1977).

[14] Tomasetta, L. R., Law, H. D., Eden, R. C., Deyhimy, I. et NAKANO, K., I.E.E.E. J. Quantum Electron. 14 (1978) 800.
[15] Law, H. D., Harris, J. S., Wong, K. C. et Tomasetta, L. R. GaAs and related compounds, Institute of Physics Conference series $\mathrm{n}^{\mathrm{o}} 45$ (Institute of Physics London) 1978, p. 420.

[16] Capasso, F., Panish, M. B., Sumski, S. et Foy, P. W., Appl. Phys. Lett. 36 (1980) 165.

[17] Widmer, R., BorTfeld, D. P. et Kleinknecht, H. P., J. Cryst. Growth 6 (1970) 237.

[18] Simashkevich, A. V. et Tsiulyanu, R. L., J. Cryst. Growth 35 (1976) 269.

[19] Gautier, P., thèse de $3^{e}$ cycle, Montpellier (1978).

[20] Kamuro, S., Hamagushi, C., Fukushima, M. et Nakai, J., Solid State Electron. 14 (1971) 1183.

[21] Chevrier, J., Etienne, D., Soonckindt, L., Bresse, J.-F. et Bougnot, G., J. Cryst. Growth 38 (1977) 309.

[22] Joullie, A., Gautier, P. et Monteil, E., J. Cryst. Growth. 47 (1979) 100.

[23] Tuвota, H., Jpn. J. Appl. Phys. 2 (1963) 259.

[24] Riben, A. R. et Feucht, D. L., Int. J. Electronics 20 (1966) 583.

[25] Riben, A. R., Feucht, D. L. et Oldham, W. G., J. Electrochem. Soc. 113 (1966) 245.

[26] Colby, J. W., MAGIC IV, A new improved version of MAGIC, Proc. 6th Nat. Conf. on Electron Probe Microanalysis, Pittsburgh, Pennsylvania, EPASA, 17A-17B (1971). 